\title{
EFICÁCIA DE HERBICIDAS NO CONTROLE EM PRÉ E PÓS-EMERGÊNCIA DO BALÃOZINHO (Cardiospermum halicacabum)
}

\author{
Alexandre M. Brighenti ${ }^{1}$, Estevo S. Bortoluzi ${ }^{2}$, Fernando S. Adegas ${ }^{3}$, \\ Dionísio L. P. Gazziero ${ }^{1}$ e Elemar Voll ${ }^{1}$
}

\footnotetext{
'Embrapa Soja. Caixa Postal 231. Londrina, PR 86970-001 brighent@cnpso.embrapa.br

${ }^{2}$ Estagiário. Embrapa Soja/Universidade Federal de Goiás, Jataí, GO

3EMATER-PR. Caixa Postal 763. Londrina, PR 86001-970 adegas@enpso.embrapa.br
}

\begin{abstract}
RESUMO
O balãozinho (Cardiospermum halicacabum) é uma planta daninha infestante, principalmente em lavouras de soja nos estados do Sul do Brasil. Devido à dificuldade de separação das sementes do balãozinho das de soja, essa espécie está se espalhando com rapidez através de sementes contaminadas da cultura. É tolerante à maioria dos herbicidas, principalmente os de contato, e, em lavouras altamente infestadas, dificulta a colheita mecânica. Três experimentos foram conduzidos na Embrapa Soja, Londrina, PR, a fim de avaliar o controle do balãozinho através da aplicação de herbicidas em condições de pré e pós-emergência dessa espécie daninha. Os tratamentos mais eficazes no controle do balãozinho em condições de préemergência foram oxyfluorfen (480 g/ha), sulfentrazone (600 g/ha), acetochlor (2304 g/ha), prometryne (1600 g/ha), alachlor (3360 g/ha) e atrazine (2500 g/ha). Em aplicações em pós-emergência, os herbicidas mais eficientes, aplicados isolados ou em mistura, foram glyphosate+carfentrazone (720 g/ha e.a.+ $12 \mathrm{~g} / \mathrm{ha})$, glyphosate+flumioxazin (720 g/ha e.a.+ $25 \mathrm{~g} / \mathrm{ha})$, glyphosate+lactofen (720 g/ha e.a. + $144 \mathrm{~g} / \mathrm{ha})$ e amônio-glufosinato (400 g/ha).
\end{abstract}

Palavras-chave: controle químico, plantas daninhas, olho-de-pombo.

\section{ABSTRACT \\ Efficacy of herbicides in pre and postemergence control of balloonvine (Cardiospermum halicacabum)}

Balloonvine (Cardiospermum halicacabum) is distributed throughout South Brazil. Because of size and shape similarities, balloonvine seeds are difficult to separate mechanically from soybean seed and this weed is spreading quickly by contaminated soybean seed. It is tolerant to most herbicides, mainly to contact products and highly infested soybean areas are difficult to harvest. Three experiments were carried out at Embrapa Soybean, Londrina, Paraná State, in order to evaluate balloonvine chemical control with pre and postemergence herbicide applications. Oxyfluorfen ( $480 \mathrm{~g} / \mathrm{ha})$, sulfentrazone (600 $\mathrm{g} / \mathrm{ha}$ ), acetochlor (2304 g/ha), prometryne (1600 g/ha), alachlor (3360 g/ha), and atrazine (2500 g/ha) were efficient on preermergence balloonvine control. Glyphosate plus carfentrazone (720 g/ha a.e $+12 \mathrm{~g} / \mathrm{ha})$, glyphosate plus flumioxazin (720 $\mathrm{g} / \mathrm{ha}$ a.e. $+25 \mathrm{~g} / \mathrm{ha})$, glyphosate plus lactofen $(720 \mathrm{~g} / \mathrm{ha}$ a.e $+144 \mathrm{~g} / \mathrm{ha})$ and glufosinate-ammonium (400 g/ha) were efficient on postemergence balloonvine control.

Key words: chemical control, weeds, heartseed. 


\section{INTRODUÇÃO}

O balãozinho é uma espécie da família Sapindaceae, originária da Ásia (Lorenzi, 2000). É uma planta anual de reprodução por sementes, ocorrendo em regiões tropicais e subtropicais do mundo (Kissmann \& Groth, 1995). Sua germinação ocorre em fluxos durante a primavera e o verão, dificultando o controle, tanto por capinas como por herbicidas. Essa espécie possui hábito trepador, com ramos e gavinhas que se enroscam em obstáculos. No seu ambiente natural, é rara a ocorrência de altas infestações, mas em lavouras podem ocorrer povoamentos consideráveis. Quando cortada acima do primeiro nó cotiledonar, a planta rebrota e essa regeneração se dá pelo desenvolvimento dos ramos axilares, em conseqüência da quebra da dominância apical (Machado et al., 1997).

As sementes da soja e do balãozinho são de difícil separação, pois ambas possuem forma arredondada, aproximadamente a mesma massa e, nas cultivares de sementes miúdas, assemelham-se em diâmetro. No Brasil, o problema é crescente e tende a se agravar pela distribuição de sementes contaminadas. Sua ocorrência vem aumentando significativamente nas lavouras de soja nos estados do Rio Grande do Sul, Paraná e Santa Catarina (Voll et al., 1998).

Como o ciclo dessa infestante é, geralmente, mais longo que o das culturas anuais, torna-se difícil a colheita em áreas altamente infestadas. Infestações da ordem de 10 plantas $/ \mathrm{m}^{2}$ podem reduzir o rendimento de grãos de soja em torno de 25,5\% (Souza e Machado, 1997). Além de reduzir a produtividade da soja, a invasora afeta também a qualidade do óleo e do farelo. Plantas de balãozinho envolvem várias outras plantas daninhas e também as da cultura, formando um emaranhado resistente à tração, dificultando o trabalho das colhedoras. As sementes dessa invasora são duras e o máximo de germinação ocorre quando se realiza a escarificação por 30 minutos em $\mathrm{H}_{2} \mathrm{SO}_{4}$ (Heit, 1974).

O objetivo deste trabalho foi avaliar o controle do balãozinho por meio da aplicação de herbicidas em condições de pré e pós-emergência dessa espécie daninha.

\section{MATERIAL E MÉTODOS}

\section{a) Controle químico do balãozinho em condições de pré- emergência}

Dois experimentos foram conduzidos em casa-de-vegetação, na Embrapa Soja, Londrina, PR. O primeiro foi instalado em 8 de agosto e o segundo em 1 de outubro de 2001. O material utilizado para enchimento dos vasos foi um solo classificado como Latossolo Roxo distrófico. Cada parcela foi constituída de um vaso de $2 \mathrm{~L}$ de capacidade, recebendo dez sementes de balãozinho. O delineamento experimental foi inteiramente casualizado com quatro repetições (primeiro experimento) e sete repetições (segundo experimento). Os tratamentos utilizados no primeiro experimento foram: imazaquin $140 \mathrm{~g} / \mathrm{ha}$ (Scepter $70 \mathrm{DG}, 200 \mathrm{~g} / \mathrm{ha}$ ), oxyfluorfen $480 \mathrm{~g} / \mathrm{ha}$ (Goal BR, 2,0 L /ha), sulfentrazone $600 \mathrm{~g} / \mathrm{ha}$ (Boral 500, 1,2 L/ha), acetochlor $2304 \mathrm{~g} /$ ha (Surpass, 3,0 L/ha), prometryne $1600 \mathrm{~g} / \mathrm{ha}$ (Prometrex, 2,0 kg/ha), alachlor 3360 $\mathrm{g} / \mathrm{ha}$ (Laço, 7,0 L/ha), diflufenican $25 \mathrm{~g} / \mathrm{ha}$ (Brodal, $500 \mathrm{~kg} /$ ha), atrazine $2500 \mathrm{~g} / \mathrm{ha}$ (Gesaprim 500, 5,0 L/ha) e a testemunha sem aplicação. No segundo experimento foram aplicados os mesmos tratamentos e doses mencionados, à exceção do tratamento com diflufenican.

A aplicação dos herbicidas foi realizada no mesmo dia da semeadura, utilizando-se um pulverizador costal, equipado com um bico de jato plano $8002 \mathrm{VS}$, com volume de calda equivalente a $180 \mathrm{~L} / \mathrm{ha}$, à pressão constante de 2,09 $\mathrm{kgf} / \mathrm{cm}^{2}$, mantida por $\mathrm{CO}_{2}$ comprimido. Por ocasião da aplicação dos produtos, a temperatura ambiente era de $25,6^{\circ} \mathrm{C}$ e $28,8^{\circ} \mathrm{C}$ e a umidade relativa do ar de $65 \%$ e $62 \%$ para o primeiro e segundo experimentos, respectivamente.

O controle foi avaliado através de escala percentual aos 12, 15 e 20 dias após a aplicação (DAA) dos herbicidas (primeiro experimento) e 15, 19 e 23 DAA dos tratamentos (segundo experimento). O valor $0 \%$ (zero) correspondeu a nenhum controle e $100 \%$ à morte de plantas. Aos 20 DAA (primeiro experimento), as plantas foram colhidas e lavadas em água corrente, colocadas em estufa de ventilação forçada de ar a $70^{\circ} \mathrm{C}$ durante 72 horas e obtidos os valores médios da biomassa seca de plantas de cada vaso. Os dados foram submetidos à análise de variância e as médias comparadas pelo teste de Tukey a $5 \%$ de probabilidade.

\section{b) Controle químico do balãozinho em condições de pós- emergência \\ O experimento foi conduzido na Embrapa Soja, Lon-} drina, PR, durante o período de 27 de setembro a 07 de dezembro de 2001. Sementes de balãozinho foram colocadas em vasos de $10 \mathrm{~L}$ de capacidade e mantidos sobre bancadas em condições de campo. O material utilizado para enchimento dos vasos foi composto da mistura de três partes de solo para uma de húmus. O delineamento experimental foi inteiramente casualizado com quatro repetições. Os tratamentos utilizados foram constituídos de vários herbicidas, de misturas de herbicidas (Tabela 1) e da testemunha sem aplicação.

Foi realizado o desbaste de plantas após a emergência do balãozinho, mantendo-se quatro plantas por vaso. A aplicação dos herbicidas foi realizada no dia 16 de novembro, correspondente a 50 dias após a semeadura. Nessa época, as plantas encontravam-se com valores médios do número de ramos e altura correspondentes a 4 e $70 \mathrm{~cm}$, respectivamente. Para aplicação dos herbicidas, foi utilizado pulverizador costal, equipado com um bico de jato plano Magno BD 110 015 , com volume de calda equivalente a $152 \mathrm{~L} /$ ha à pressão constante de $2,22 \mathrm{kgf} / \mathrm{cm}^{2}$, mantida por $\mathrm{CO}_{2}$ comprimido. Por ocasião da aplicação dos produtos, a temperatura ambiente era de $25^{\circ} \mathrm{C}$ e a umidade relativa do ar de $65 \%$. 
Tabela 1. Características dos herbicidas que fizeram parte dos tratamentos aplicados em condições de pós-emergência do balãozinho. Embrapa Soja, Londrina, PR, 2001.

\begin{tabular}{|c|c|c|c|c|}
\hline \multicolumn{2}{|c|}{ Herbicida } & \multirow[t]{2}{*}{ Concentração } & \multicolumn{2}{|c|}{$\operatorname{Dose}^{(1)}$} \\
\hline Nome técnico & Nome comercial & & $\begin{array}{l}\text { (g/hai.a. }{ }^{\left({ }^{1}\right)} \\
\left.\text { ou e.a. }{ }^{\left({ }^{(}\right)}\right)\end{array}$ & $\begin{array}{c}\text { (g ou mL/ha } \\
\left.\text { p.c. }{ }^{(3)}\right)\end{array}$ \\
\hline Imazapyr & Arsenal 250 & $250 \mathrm{~g} / \mathrm{L}$ & 50 & 200 \\
\hline Imazapyr & Arsenal 250 & $250 \mathrm{~g} / \mathrm{L}$ & 100 & 400 \\
\hline Foransulfuron / iodosulfuron & Equip Plus ${ }^{(4)}$ & $300 \mathrm{~g} / \mathrm{kg} / 20 \mathrm{~g} / \mathrm{kg}$ & $45 / 3$ & 150 \\
\hline Glyphosate & Round up WG ${ }^{(5)}$ & $720 \mathrm{~g} / \mathrm{L}$ & 720 & 1000 \\
\hline Glyphosate + carfentrazone & Round up WG + Aurora ${ }^{(5)}$ & $720 \mathrm{~g} / \mathrm{L}+400 \mathrm{~g} / \mathrm{L}$ & $720+12$ & $1000+30$ \\
\hline Glyphosate + flumioxazin & Round up WG + Flumyzin ${ }^{(5)}$ & $720 \mathrm{~g} / \mathrm{L}+500 \mathrm{~g} / \mathrm{kg}$ & $720+25$ & $1000+50$ \\
\hline Gliphosate + lactofen & Round up WG + Cobra & $720 \mathrm{~g} / \mathrm{L}+240 \mathrm{~g} / \mathrm{L}$ & $720+144$ & $1000+600$ \\
\hline Cloransulam & Pacto & $840 \mathrm{~g} / \mathrm{kg}$ & 39,4 & 47 \\
\hline Cloransulam + lactofen & Pacto + Cobra ${ }^{(6)}$ & $840 \mathrm{~g} / \mathrm{kg}+240 \mathrm{~g} / \mathrm{L}$ & $39,4+144$ & $47+600$ \\
\hline Cloransulam + carfentrazone & Pacto + Aurora $a^{(6)}$ & $840 \mathrm{~g} / \mathrm{kg}+400 \mathrm{~g} / \mathrm{L}$ & $39,4+12$ & $47+30$ \\
\hline Cloransulam + flumioxazin & Pacto + Flumyzin ${ }^{(6)}$ & $840 \mathrm{~g} / \mathrm{kg}+500 \mathrm{~g} / \mathrm{kg}$ & $39,4+25$ & $47+50$ \\
\hline Lactofen & Cobra & $240 \mathrm{~g} / \mathrm{L}$ & 144 & 600 \\
\hline Carfentrazone & Aurora & $400 \mathrm{~g} / \mathrm{L}$ & 12 & 30 \\
\hline Flumioxazin & Flumyzin & $500 \mathrm{~g} / \mathrm{kg}$ & 25 & 50 \\
\hline Fomesafen & Flex & $250 \mathrm{~g} / \mathrm{L}$ & 300 & 1200 \\
\hline Lactofen + carfentrazone & Cobra + Aurora & $240 \mathrm{~g} / \mathrm{L}+400 \mathrm{~g} / \mathrm{L}$ & $144+12$ & $600+30$ \\
\hline Lactofen + flumioxazin & Cobra + Flumyzin & $240 \mathrm{~g} / \mathrm{L}+500 \mathrm{~g} / \mathrm{kg}$ & $144+25$ & $600+50$ \\
\hline Carfentrazone + flumioxazin & Aurora + Flumyzin & $400 \mathrm{~g} / \mathrm{L}+500 \mathrm{~g} / \mathrm{kg}$ & $12+25$ & $30+50$ \\
\hline Lactofen + fomesafen & Cobra + Flex ${ }^{(7)}$ & $240 \mathrm{~g} / \mathrm{L}+250 \mathrm{~g} / \mathrm{L}$ & $144+300$ & $600+1200$ \\
\hline Amônio-glufosinato & Finale $e^{(8)}$ & $200 \mathrm{~g} / \mathrm{L}$ & 400 & 2000 \\
\hline
\end{tabular}

(1)i.a. (ingrediente ativo), (2)e.a. (equivalente acido), ${ }^{(3)}$ p.c. (produto comercial).

${ }^{(4)}+$ Herbitensil 0,5\% v/v, ${ }^{(5)}+$ Assist 0,5\% v/v, ${ }^{(6)}+$ Agral 0,2\% v/v, ${ }^{(7)}+$ Energic 0,5\% v/v, ${ }^{(8)}+$ Herbitensil $0,2 \% \mathrm{v} / \mathrm{v}$.

O controle foi avaliado através de escala percentual aos 3, 7, 12 e 21 dias após a aplicação dos tratamentos, onde $0 \%$ (zero) correspondeu a nenhum controle e $100 \%$ à morte de plantas. Aos 21 DAA, as plantas foram colhidas e lavadas em água corrente, colocadas em estufa de ventilação forçada de ar a $70{ }^{\circ} \mathrm{C}$ durante 72 horas e obtido os valores médios de biomassa seca. Os dados foram submetidos à análise de variância e as médias comparadas pelo teste de Tukey a 5\% de probabilidade.

\section{RESULTADOS E DISCUSSÃO}

\section{a) Controle do balãozinho em condições de pré-emer- gência}

Os herbicidas oxyfluorfen, sulfentrazone, acetochlor, prometryne, alachlor e atrazine foram eficazes no controle do balãozinho, alcançando valores acima de $80 \%$, aos 20 DAA (Tabela 2). Apenas o imazaquin e o diflufenican foram pouco eficazes no controle do balãozinho, obtendo-se percentagens de 35 e $18 \%$ aos 20 DAA, respectivamente. Com relação à biomassa seca, os tratamentos com os herbicidas oxyfluorfen, sulfentrazone, acetochlor, prometryne, alachlor e atrazine resultaram em valores mais baixos para essa característica, diferindo da testemunha sem aplicação. Esses resultados são importantes quando se adota o binômio soja/ milho. A utilização de herbicidas como o sulfentrazone na soja e o acetochlor, o alachlor e o atrazine no milho são práticas importantes no manejo de áreas infestadas com essa espécie.

Os tratamentos mais eficazes no controle do balãozinho, aos 23 dias também foram oxyfluorfen, sulfentrazone, acetochlor, prometryne, alachlor e atrazine (Tabela 3). Todos eles apresentaram controle acima de $88 \%$, exceto o imazaquin, confirmando os resultados do experimento anterior.

\section{b) Controle do balãozinho em condições de pós-emergên} cia

Aos 21 DAA as misturas de glyphosate mais carfentrazone, gliphosate mais flumioxazin, glyphosate mais lactofen e o herbicida amônio-glufosinato foram eficazes no controle do balãozinho, apresentando percentagens de 88 , 94, 96 e 92\%, respectivamente (Tabela 4). O glyphosate aplicado isoladamente apresentou controle mediano aos 21 DAA 
Tabela 2. Efeitos de herbicidas aplicados em pré-emergência do balãozinho (Primeiro experimento). Embrapa Soja, Londrina, PR, 2001.

\begin{tabular}{|c|c|c|c|c|c|}
\hline \multirow{2}{*}{ Tratamento } & \multirow{2}{*}{$\begin{array}{c}\text { Dose } \\
(\mathrm{g} / \mathrm{h} a)\end{array}$} & \multicolumn{3}{|c|}{ Controle $(\%)$} & \multirow{2}{*}{$\begin{array}{c}\text { Biomassa seca } \\
\text { (g/planta) } \\
\text { 20DAA }\end{array}$} \\
\hline & & 12 DAA & 15 DAA & 20 DAA & \\
\hline Imazaquin & 140 & $45 d^{1}$ & $38 \mathrm{~b}$ & $35 \mathrm{~b}$ & $0,0254 \mathrm{abc}$ \\
\hline Oxyfluorfen & 480 & $88 \mathrm{a}$ & $94 \mathrm{a}$ & $94 \mathrm{a}$ & $0,0123 \mathrm{c}$ \\
\hline Sulfentrazone & 600 & $95 \mathrm{a}$ & $88 \mathrm{a}$ & $84 \mathrm{a}$ & $0,0187 \mathrm{bc}$ \\
\hline Acetochlor & 2304 & $78 \mathrm{ab}$ & $86 \mathrm{a}$ & $89 \mathrm{a}$ & $0,0153 \mathrm{c}$ \\
\hline Prometryne & 1600 & $66 \mathrm{bc}$ & $89 \mathrm{a}$ & $91 \mathrm{a}$ & $0,0184 \mathrm{bc}$ \\
\hline Alachlor & 3360 & $81 \mathrm{ab}$ & $91 \mathrm{a}$ & $90 \mathrm{a}$ & $0,0172 \mathrm{bc}$ \\
\hline Diflufenican & 25 & $20 \mathrm{e}$ & $25 \mathrm{bc}$ & $18 \mathrm{bc}$ & $0,0392 \mathrm{ab}$ \\
\hline Atrazine & 2500 & $55 \mathrm{~cd}$ & $90 \mathrm{a}$ & $91 \mathrm{a}$ & $0,0149 \mathrm{c}$ \\
\hline Testemunha sem aplicação & - & $0 \mathrm{f}$ & $0 \mathrm{c}$ & $0 \mathrm{c}$ & 0,0443 a \\
\hline DMS (5\%) & & 17,5 & 27,8 & 29,3 & 0,0278 \\
\hline CV $(\%)$ & - & 12,4 & 17,3 & 18,6 & 41,8 \\
\hline
\end{tabular}

(1)Médias seguidas pelas mesmas letras na coluna não diferem entre si pelo teste de Tukey a $5 \%$ de probabilidade.

Tabela 3. Efeito de herbicidas aplicados em pré-emergência do balãozinho (Segundo experimento). Embrapa Soja, Londrina, PR, 2001.

\begin{tabular}{lcccc}
\hline \multirow{2}{*}{\multicolumn{1}{c}{ Tratamento }} & Dose & \multicolumn{3}{c}{ Controle (\%) } \\
\cline { 3 - 5 } & $(\mathbf{g / h a})$ & $\mathbf{1 5} \mathbf{~ D A A}$ & 19 DAA & 23 DAA \\
\hline Imazaquin & 140 & $17 \mathrm{c}^{1}$ & $16 \mathrm{~b}$ & $18 \mathrm{c}$ \\
Oxyfluorfen & 480 & $92 \mathrm{a}$ & $93 \mathrm{a}$ & $99 \mathrm{ab}$ \\
Sulfentrazone & 600 & $100 \mathrm{a}$ & $100 \mathrm{a}$ & $100 \mathrm{a}$ \\
Acetochlor & 2304 & $93 \mathrm{a}$ & $100 \mathrm{a}$ & $100 \mathrm{a}$ \\
Prometryne & 1600 & $28 \mathrm{c}$ & $94 \mathrm{a}$ & $98 \mathrm{ab}$ \\
Alachlor & 3360 & $96 \mathrm{a}$ & $99 \mathrm{a}$ & $100 \mathrm{a}$ \\
Atrazine & 2500 & $41 \mathrm{~b}$ & $87 \mathrm{a}$ & $88 \mathrm{~b}$ \\
Testemunha sem aplicação & - & $0 \mathrm{~d}$ & $0 \mathrm{c}$ & $0 \mathrm{~d}$ \\
\hline DMS (5\%) & - & 11,9 & 13,4 & 10,9 \\
CV (\%) & - & 11,9 & 10,7 & 8,4 \\
\hline
\end{tabular}

${ }^{1}$ Médias seguidas pelas mesmas letras na coluna não diferem entre si pelo teste de Tukey a $5 \%$ de probabilidade.

$(72 \%)$ e os demais tratamentos foram pouco eficazes no controle dessa espécie daninha. O efeito da aplicação de diversos herbicidas utilizados isolados ou em mistura foi avaliado por Souza e Machado (1997). Aplicaram os tratamentos em pósemergência precoce da cultura da soja e verificaram que os tratamentos com lactofen (180 g/ha), fomesafen ( $250 \mathrm{~g} / \mathrm{ha}) \mathrm{e}$ a mistura lactofen mais fomesafen $(120+125 \mathrm{~g} / \mathrm{ha})$ controlaram o balãozinho, obtendo percentagens que variaram de 70 a $90 \%$. Esse resultado não foi confirmado neste experimento, pois esses mesmos tratamentos resultaram em percentagens de controle abaixo de $60 \%$. Provavelmente, esses valores mais baixos ocorreram, em função do balãozinho estar em um estádio mais avançado na época de aplicação dos produtos. $\mathrm{E}$ as folhas superiores, cobrindo as inferiores (efeito "guarda-chuva"), evitaram que esses herbicidas de contato atingissem completamente a planta daninha.
Com relação à biomassa seca, os tratamentos com os herbicidas glyphosate mais carfentrazone, glyphosate mais flumioxazin, glyphosate mais lactofen e amônio-glufosinato resultaram em valores mais baixos para essa característica, diferindo da testemunha sem aplicação.

Em lavouras de soja transgênica tolerante ao glyphosate, aplicações desse herbicida em mistura com carfentrazone, flumioxazin ou lactofen, nas doses testadas neste trabalho são, provavelmente, práticas viáveis no controle do balãozinho. Existe ainda a recomendação de uso do herbicida amônio-glufosinato em dessecação da cultura da soja (Rodrigues e Almeida, 1998). Assim, lavouras que, no momento da colheita, encontram-se infestadas com balãozinho, a aplicação desse herbicida proporcionará controle eficiente dessa espécie, facilitando a operação de colheita. 
Eficácia de herbicidas no controle do balãozinho

Tabela 4. Efeito de herbicidas aplicados em pós-emergência do balãozinho. Embrapa Soja, Londrina, PR, 2001.

\begin{tabular}{|c|c|c|c|c|c|c|}
\hline \multirow{2}{*}{ Tratamento } & \multirow{2}{*}{$\begin{array}{c}\text { Dose } \\
\text { (g/ha) }\end{array}$} & \multicolumn{4}{|c|}{ Controle (\%) } & \multirow{2}{*}{$\begin{array}{c}\text { Biomassa seca } \\
\text { (g/planta) } \\
21 \text { DAA }\end{array}$} \\
\hline & & $3 \mathrm{DAA}$ & $7 \mathrm{DAA}$ & $12 \mathrm{DAA}$ & $21 \mathrm{DAA}$ & \\
\hline Imazapyr & 50 & $5 \mathrm{ij}^{1}$ & $10 \mathrm{gh}$ & $15 \mathrm{i}$ & $15 \mathrm{i}$ & $8,63 \mathrm{bcd}$ \\
\hline Imazapyr & 100 & $10 \mathrm{hi}$ & $15 \mathrm{~g}$ & $25 \mathrm{~h}$ & $40 \mathrm{~g}$ & 7,99 cde \\
\hline Foransulfur on + iodosulfuron & $45+3$ & $5 \mathrm{ij}$ & $5 \mathrm{hi}$ & $10 \mathrm{ij}$ & $10 \mathrm{ij}$ & $11,69 \mathrm{ab}$ \\
\hline Glyphosate & 720 & $12 \mathrm{~h}$ & $32 \mathrm{e}$ & $68 \mathrm{bc}$ & $72 \mathrm{c}$ & 3,28 fgh \\
\hline Glyphosate + carfentrazone & $720+12$ & $49 \mathrm{~d}$ & $52 \mathrm{c}$ & $72 \mathrm{~b}$ & $88 \mathrm{~b}$ & 3,28 fgh \\
\hline Glyphosate + flumioxazin & $720+25$ & $80 \mathrm{a}$ & $86 \mathrm{a}$ & $90 \mathrm{a}$ & $94 \mathrm{a}$ & $2,16 \mathrm{~h}$ \\
\hline Glyphosate + lactofen & $720+144$ & $80 \mathrm{a}$ & $81 \mathrm{a}$ & 92 a & $96 \mathrm{a}$ & $2,30 \mathrm{~h}$ \\
\hline Cloransulam & 39,4 & $5 \mathrm{ij}$ & $5 \mathrm{hi}$ & $5 \mathrm{jk}$ & $5 \mathrm{jk}$ & $11,00 \mathrm{abc}$ \\
\hline Cloransulam + lactofen & $39,4+144$ & $62 \mathrm{bc}$ & $64 \mathrm{~b}$ & $69 \mathrm{bc}$ & $62 d$ & 4,69 efgh \\
\hline Cloransulam + carfentrazone & $39,4+12$ & $35 \mathrm{f}$ & $35 \mathrm{e}$ & $36 \mathrm{~g}$ & $38 \mathrm{~g}$ & $8,86 \mathrm{abcd}$ \\
\hline Cloransulam + flumioxazin & $39,4+25$ & $51 \mathrm{~d}$ & $51 \mathrm{c}$ & $68 \mathrm{bc}$ & $58 \mathrm{de}$ & 5,91 defg \\
\hline Lactofen & 144 & $59 \mathrm{bc}$ & $61 \mathrm{~b}$ & $66 \mathrm{c}$ & $58 \mathrm{de}$ & 5,77 defg \\
\hline Carfentrazone & 12 & $30 \mathrm{fg}$ & 30 ef & $30 \mathrm{~h}$ & $25 \mathrm{~h}$ & 12,17 a \\
\hline Flumioxazin & 25 & $26 \mathrm{~g}$ & $26 \mathrm{f}$ & $52 \mathrm{e}$ & 52 ef & 5,97 defg \\
\hline Fomesafen & 300 & $42 \mathrm{e}$ & $42 \mathrm{~d}$ & $42 \mathrm{f}$ & $25 \mathrm{~h}$ & $10,03 \mathrm{abc}$ \\
\hline Lactofen + carfentrazone & $144+12$ & $57 \mathrm{c}$ & $59 \mathrm{~b}$ & $59 \mathrm{~d}$ & $51 \mathrm{f}$ & 5,83 defg \\
\hline Lactofen + flumioxazin & $144+25$ & $64 \mathrm{~b}$ & $64 \mathrm{~b}$ & $68 \mathrm{bc}$ & $58 \mathrm{de}$ & $6,38 \mathrm{def}$ \\
\hline Carfentrazone + flumioxazin & $12+25$ & $49 \mathrm{~d}$ & $49 c$ & $65 c$ & 54 ef & 5,18 efgh \\
\hline Lactofen + fomesafen & $144+300$ & $61 \mathrm{bc}$ & $61 \mathrm{~b}$ & $65 c$ & $58 \mathrm{de}$ & 5,18 efgh \\
\hline Amônio-glufosinato & 400 & $31 \mathrm{fg}$ & $85 \mathrm{a}$ & $91 \mathrm{a}$ & $92 \mathrm{ab}$ & $2,82 \mathrm{gh}$ \\
\hline Testemunha sem aplicação & - & $0 \mathrm{j}$ & $0 \mathrm{i}$ & $0 \mathrm{k}$ & $0 \mathrm{k}$ & $11,72 \mathrm{ab}$ \\
\hline DMS (5\%) & - & 5,2 & 5,3 & 5,4 & 5,9 & 3,3 \\
\hline CV $(\%)$ & - & 5,1 & 4,6 & 3,9 & 4,5 & 18,9 \\
\hline
\end{tabular}

${ }^{1}$ Médias seguidas pelas mesmas letras na coluna não diferem entre si pelo teste de Tukey a $5 \%$ de probabilidade.

\section{CONCLUSÕES}

Os herbicidas mais eficazes no controle do balãozinho em condições de pré-emergência foram oxyfluorfen $(480 \mathrm{~g} /$ ha), sulfentrazone (600 g/ha), acetochlor (2304 g/ha), prometrine $(1600 \mathrm{~g} / \mathrm{ha})$, alachlor $(3360 \mathrm{~g} / \mathrm{ha})$ e atrazine $(2500$ $\mathrm{g} / \mathrm{ha}$ ). Em aplicações em pós-emergência dessa planta daninha, os herbicidas mais eficazes, aplicados isolados ou em mistura, foram glyphosate mais carfentrazone (720 g/ha e.a. $+12 \mathrm{~g} / \mathrm{ha}$ ), glyphosate mais flumioxazin (720 g/ha e.a. +25 $\mathrm{g} / \mathrm{ha})$, glyphosate mais lactofen (720 g/ha e.a. $+144 \mathrm{~g} / \mathrm{ha}) \mathrm{e}$ amônio-glufosinato (400 g/ha).

\section{LITERATURA CITADA}

HEIT, C.E. Germination and hard seeds studies with $C$. halicacabum (balloonvine, heartseed) in laboratory testing. Newsletter of the Association Official Seed Analysts, Fort Collins, v.48, p.35-37, 1974.

KISSMANN, K.G; GROTH, D. Plantas infestantes e nocivas. São Paulo: BASF, 1995. t.2, 683 p.
LORENZI, H. Plantas daninhas do Brasil: terrestres, aquáticas, parasitas, tóxicas e medicinais. 3 ed. Nova Odessa: Plantarum, 2000. 608 p.

MACHADO, S.L.O.; ÁVILA, L.A.; SOUZA, R.O.; SCHADECK, F.A.; CASSOL, I.A.B. Efeito da época e intensidade de corte na regeneração adventícia do olhode-pombo (Cardiospermum halicacabum). In: CONGRESSO BRASILEIRO DA CIÊNCIA DAS PLANTAS DANINHAS, 21. Caxambu, 1997. Resumos... Viçosa: Sociedade Brasileira da Ciência das Plantas Daninhas, 1997. p.12.

RODRIGUES, B.N.; ALMEIDA, F.S. Guia de herbicidas. 4 ed. Londrina, PR: Edição dos Autores, 1998. 648 p.

SOUZA, R.O.; MACHADO, S.L.O. Controle químico do olho-de-pombo (Cardiospermum halicacabum) na cultura da soja. In: CONGRESSO BRASILEIRO DA CIENCIA DAS PLANTAS DANINHAS, 21. Caxambu, 1997. Resumos... Viçosa: Sociedade Brasileira da Ciência das Plantas Daninhas, 1997. p.137 
Alexandre M. Brighenti et al.

VOLL, E.; GAZZIERO, D.L.P.; ADEGAS, F.S.; BRIGHENTI, A.M. Dinâmica do estabelecimento e competição do balãozinho. In: REUNIÃO DE PESQUISA DE SOJA DA REGIÃO SUL, 26. Cruz Alta, 1998. Ata e resumos... Cruz Alta: UNICRUZ, 1998. p.141. 